\title{
A Stark-tuned laser application for interferometry and polarimetry on the National Spherical Torus Experiment
}

\author{
K. C. Lee, ${ }^{\text {a) }}$ C. W. Domier, B. H. Deng, M. Johnson, B. R. Nathan, \\ and N. C. Luhmann, Jr. \\ University of California at Davis, Davis, California 95616 \\ H. Park \\ Princeton Plasma Physics Laboratory, Princeton, New Jersey 08543
}

(Presented on 8 July 2002)

\begin{abstract}
Interferometer/polarimeter systems based on far infrared lasers have suffered from lack of frequency modulation techniques that can allow higher intermediate frequency (IF) frequency for a fast time resolution together with stable operation including low frequency drift. This is mainly due to the intrinsic narrow gain profile of the lasers. It is known that the Stark effect can broaden the laser gain profile as has been demonstrated in the laboratory. A Stark-tuned optically pumped far infrared (FIR) $\mathrm{CH}_{3} \mathrm{OH}$ laser together with two additional FIR lasers at $119 \mu \mathrm{m}$ have been successfully implemented in the far infrared tangential interferometer/polarimeter system which will provide temporally and radially resolved two-dimensional electron density profile $\left[n_{e}(r, t)\right]$ and toroidal field profile $\left[B_{T}(r, t)\right]$ data for the National Spherical Tokamak Experiment. The characteristic frequencies of the IF system are $\sim 3, \sim 4$, and $\sim 7 \mathrm{MHz}$ and a phase lock system was utilized for tracking the drift of the IF frequencies. In this article, the characteristics and operation of the Stark-tuned laser are described in detail. The measured electron density and Faraday rotation for various physics operation regimes are compared to the line integral of Thomson scattering measurement and EFIT results, respectively. (C) 2003 American Institute of Physics.
\end{abstract} [DOI: $10.1063 / 1.1530352]$

\section{INTRODUCTION}

Thus far, several methods of modulation frequency generation have been developed for use in heterodyne far infrared (FIR) laser interferometry. Two of the most well known techniques are mechanical rotation of gratings and two-laser systems with slightly detuned cavities. The maximum achievable frequency shift of the rotating grating method is less than $100 \mathrm{kHz}$ and that of the two-laser method is less than $2 \mathrm{MHz}$ because they are limited by the speed of the mechanical rotation and the intrinsic narrow gain bandwidth available in the FIR lasers, respectively. In principle, heterodyne interferometry measures the phase change between two sinusoidal beat frequencies: the reference signal of $\cos \left(\omega_{i f} t\right)$ and the plasma signal of $\cos \left[\omega_{i f} t+\phi(t)\right]$. Here, $\omega_{i f}$ is the modulation frequency and $\phi(t)$ is the phase change proportional to the plasma density. In the case of rapid changes in density such as plasma disruptions, if $d \phi(t) / d t$ is so large that the frequency of the plasma signal exceeds the bandwidth of the fringe counting system (that is limited by the modulation frequency itself), then the loss of fringe count will occur. Therefore, the following condition must be satisfied:

$$
\frac{d \phi(t)}{d t} \ll \omega_{i f} .
$$

Consequently, if the modulation frequency is increased, the

${ }^{\text {a)} E l e c t r o n i c ~ m a i l: ~ k c l e e @ p p p l . g o v ~}$ time resolution can be improved. For this purpose of increasing the modulation frequency and time resolution, the characteristics of high power Stark-tuned FIR laser were investigated by Mansfield ${ }^{1}$ and it was demonstrated that the Starktuned laser can generate a modulation frequency up to 30 $\mathrm{MHz}$ and that this scheme is more stable because the laser is operating at the peak of the gain curve rather than on the edge. This article describes an actual application of a Starktuned laser for plasma diagnostics on the National Spherical Tokamak Experiment (NSTX) ${ }^{2}$ that also utilizes Rommer's scheme $^{3}$ for the polarimetry as well as interferometry. In Secs. II and III, the principles of the far infrared tangential interferometer/polarimeter (FIReTIP) system and the characteristics of the Stark-tuned laser will be discussed. In Sec. IV, analysis of measured data from NSTX plasma operation during the 2001 and 2002 campaigns will be presented and followed by the conclusion.

\section{PRINCIPLES}

Figure 1 is a schematic diagram of the FIReTIP system. There are three $\mathrm{CH}_{3} \mathrm{OH}$ lasers operating at $118.8 \mu \mathrm{m}$ pumped by a $\mathrm{CO}_{2}$ laser. Two of them (FIR1 and FIR2), whose frequencies are slightly different, generate lineally polarized probe beams that are orthogonal to each other. Then after combining by polarizer and passing through a quarter wave plate, one of the beams becomes right circularly polarized and the other becomes left circularly polarized. This combined beam is separated into two beams, one goes directly to the reference detector and the other goes to the 


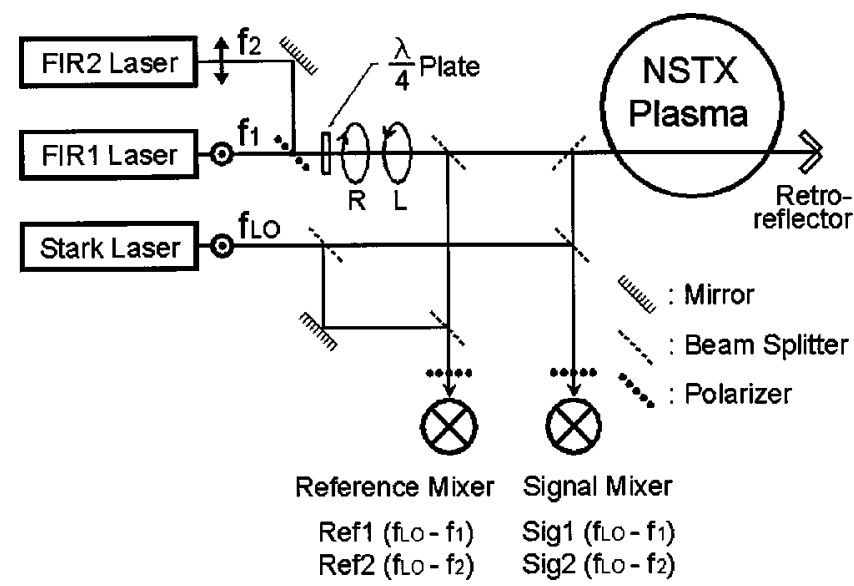

FIG. 1. Schematic diagram of FIReTIP system.

signal detector after going through the plasma and bouncing from a retroreflector. On the other hand, the third laser that is a Stark-tuned laser generates a local oscillator beam at the frequency with modulation of $5 \mathrm{MHz}$ by the Stark effect. While the probe beams traverse the plasma they experience a phase shift since the index of refraction depends on the electron density. This phase shift through the beam path in case of ordinary wave propagation is given by the following equation:

$$
\phi(x)=2.8 \times 10^{-15} \lambda \int_{0}^{x} n\left(x^{\prime}\right) d x^{\prime} .
$$

This phase shift is common to both probe beams. In the presence of a parallel magnetic field component that mainly arises from the toroidal magnetic field, $B_{T}$, the probe beams undergo Faraday rotation that makes the polarizations of the beams rotate through the beam path to an angle given by

$$
\Psi(x)=2.6 \times 10^{-13} \lambda^{2} \int_{0}^{x} n\left(x^{\prime}\right) B_{T}\left(x^{\prime}\right) d x^{\prime} .
$$

The direction of Faraday rotation for the right circularly polarized beam is opposite to that of the left circularly polarized beam. The interferometer portion of the FIReTIP system measures the phase difference between intermediate frequency (IF) signals of the reference mixer (Ref. 1 or 2) and the IF signal of the scene-beam mixer (Sig. 1 or 2) in Fig. 1 and the polarimeter measures the difference between phases of Sig. 1 and Sig. 2 that is twice the angle indicated by Eq. (3). The primary goal of the FIReTIP system on NSTX is temporally resolved two-dimensional (2D) data of plasma density and toroidal magnetic field by a multichannel configuration. The details of the FIReTIP system and its development are presented elsewhere. ${ }^{4}$

\section{CHARACTERISTICS OF STARK-TUNED LASER}

It has been demonstrated that when the gain medium is immersed in a uniform electric field, the $118.8 \mu \mathrm{m}$ line of $\mathrm{CH}_{3} \mathrm{OH}$ splits into two equivalent gain curves due to the linear splitting between sublevels of FIR transitions at low pump power ${ }^{5}$ and at high pump power. ${ }^{1}$ Figure 2 shows the design of a Stark-tuned FIR resonator that is quite similar to

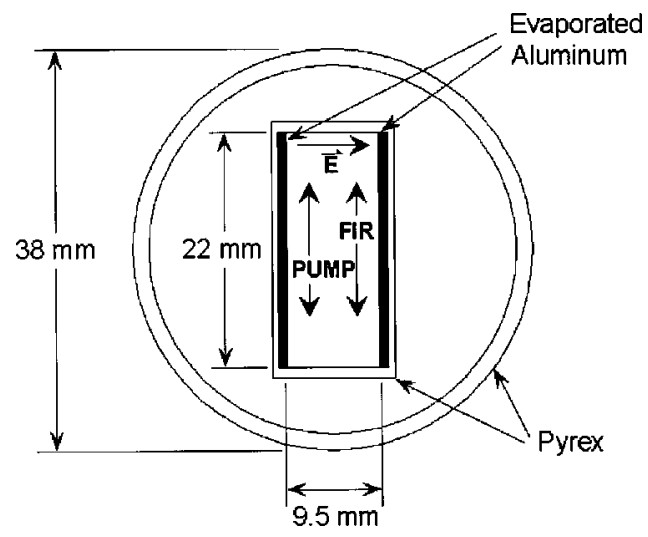

FIG. 2. Cross section of the FIR resonator tube used in the Stark-tuned laser.

the one described in Ref. 1. Two faces of the rectangular tube are coated by evaporating a conducting film of aluminum. The rectangular tube is surrounded by a cylindrical water jacket so that the entire structure is water cooled free from electrical breakdown. The input coupler consists of a flat Au-coated copper mirror with a centered $4 \mathrm{~mm}$ input coupling hole. A hybrid metal-dielectric mirror with a $6 \mathrm{~mm} \mathrm{Si}$ aperture is employed as the output coupler which is commonly used in most high power FIR laser systems. The output of the two conventional FIR lasers was $\sim 40 \mathrm{~mW}$ and that of the Stark laser was $\sim 20 \mathrm{~mW}$. The applied voltage was $\sim 400 \mathrm{~V}$ to avoid an occasional breakdown at the operating pressure ( $\sim 50 \mathrm{mT}$ at the pump side of the gas system) which was lower than the test setup used in Ref. 1 . The cavity scan of the Stark-tuned laser is shown in Fig. 3. The Stark-tuned laser was operated at one of the two shifted gain peaks so that it generates $\sim 5 \mathrm{MHz}$ modulation frequency. This spectral performance is very much consistent with the results in Ref. 1. The IF signal spectrum of the three FIR lasers' beat waves is shown in Fig. 4. The operating condition was optimized at the beat signals of $\sim 6 \pm 1 \mathrm{MHz}$ and $\sim 4 \pm 1 \mathrm{MHz}$ so that the other beat frequency between the two conventional FIR lasers is $\sim 2 \mathrm{MHz}$ as demonstrated in Fig. 4. The

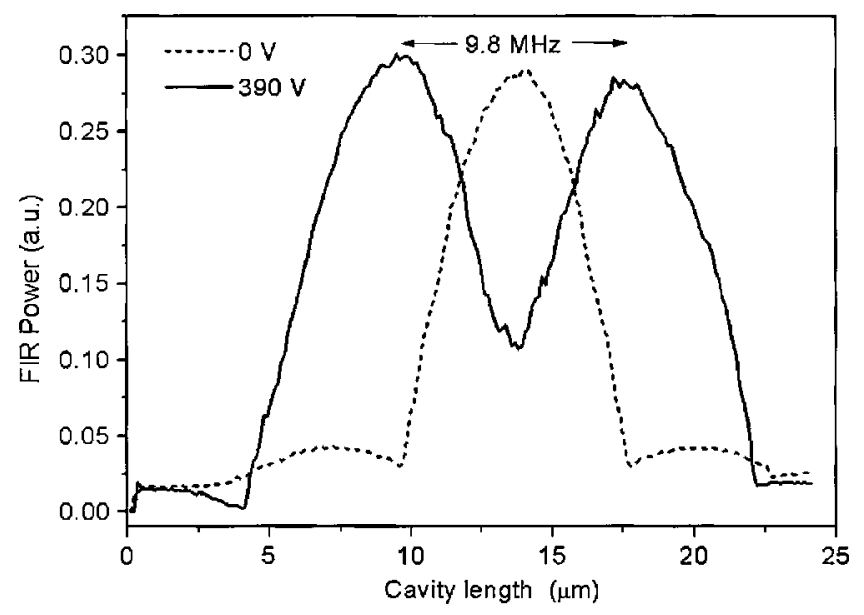

FIG. 3. Cavity scan from Stark-tuned laser with and without applied dc electric field. 


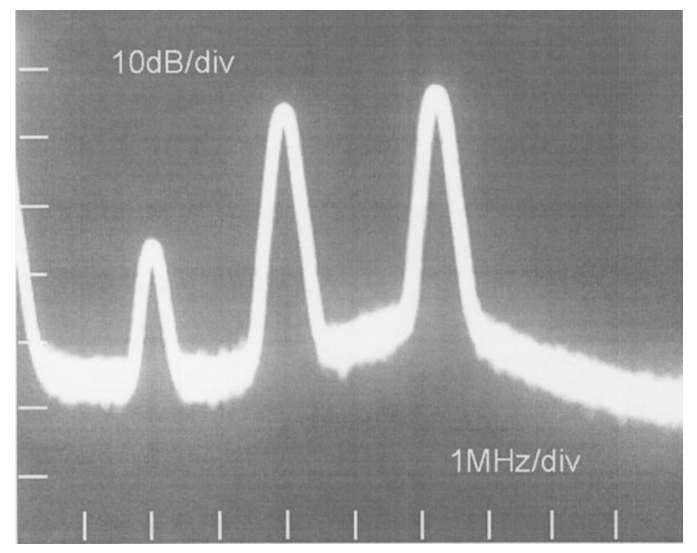

FIG. 4. Beat spectrum of three FIR lasers from the IF signal in the reference mixer (right hand side is $6 \pm 1 \mathrm{MHz}$, middle one is $4 \pm 1 \mathrm{MHz}$, and left hand side is $\sim 2 \mathrm{MHz}$ that is the beat frequency between FIR1 and FIR2).

choice of this frequency was to optimize the output power while minimizing operational risks such as electrical breakdown.

\section{EXPERIMENTAL RESULTS}

The FIReTIP system design for NSTX is based on a wavelength of $\sim 119 \mu \mathrm{m}$ that is ideal for NSTX plasma parameters. At this wavelength, the expected signal to noise ratio for interferometry and polarimetry is excellent. The interferometer system will have $\sim 10$ fringes shift and the polarimetry system will have a Faraday rotation of $\sim 10^{\circ}$ while refraction of the beam is minimum (less than $\sim 0.5 \mathrm{~mm}$ ) as shown in Fig. 3 in Ref. 2. Due to access limitations, the present system consists of seven beam lines that enter at three separate toroidal locations at Bay $\mathrm{K}$ on NSTX as shown in Fig. 5. Each beam traverses a different chord along the horizontal midplane of the device, reflects from a suitably placed corner cube retroreflector, and traverses back through the plasma to return to a common point for detec-

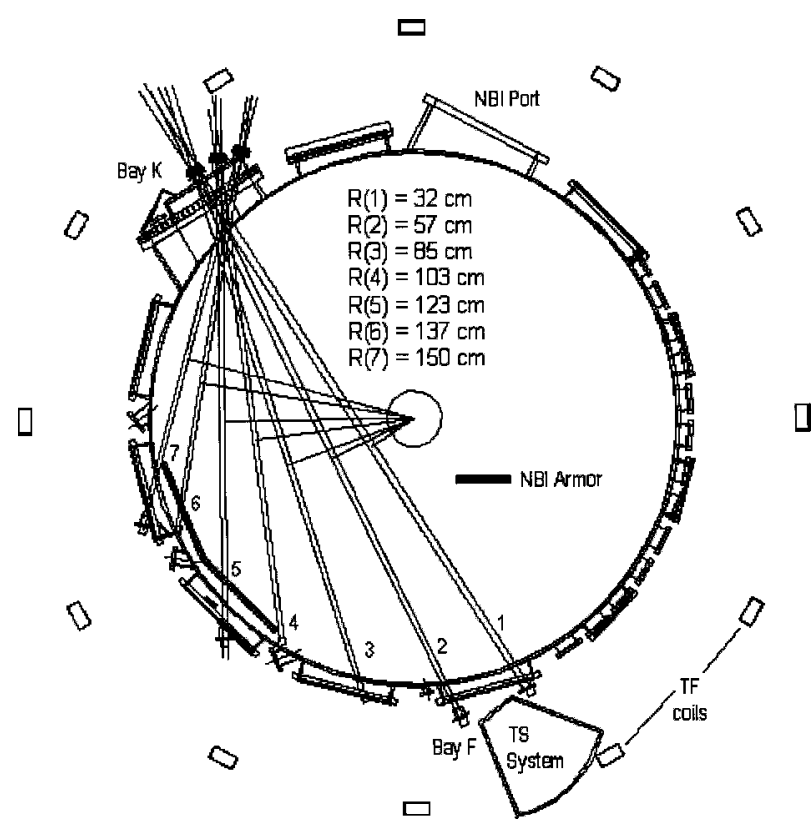

FIG. 5. Seven channel FIReTIP system will have fan view of beam lines.
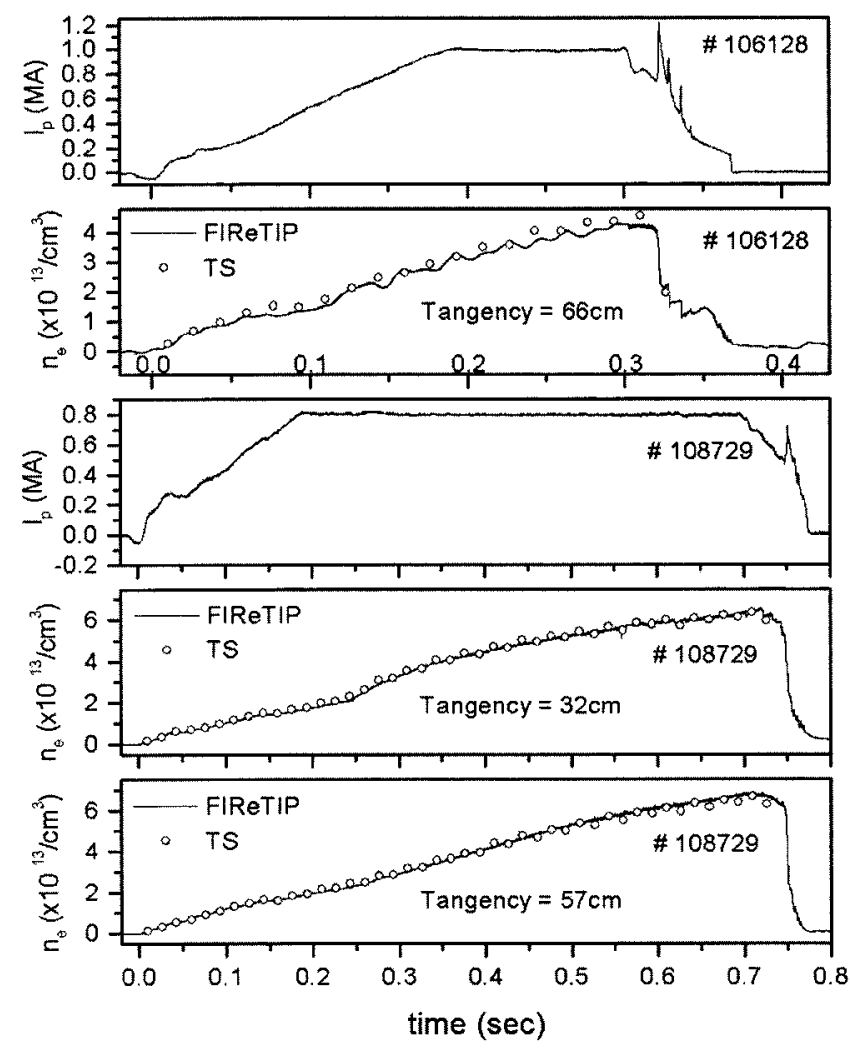

FIG. 6. Measured line averaged densities from the two chords before (10 6128) and after (10 8729) installation of vibration isolation stands are compared with the integrated value of Thomson scattering measurement along two chords.

tion. This scheme is shown schematically in this figure. During the 2001 campaign, the goal was to demonstrate a single channel interferometry capability and feasibility of Faraday rotation measurement. Since the Bay K where a temporary entrance window has been installed is scheduled to be redesigned, one channel was aligned at the tangency of $\sim 66 \mathrm{~cm}$ following the concept design of the FIReTIP system. In this campaign, a single channel operation was successful and test results of Faraday rotation measurements were acquired. However, there were numerous sources of mechanical vibrations that superposed in the measured density. During this campaign, significant effort was devoted to decouple the magnetic influence which was originated from the $\mathrm{OH}$ system. Note that the laser system was hardened in the Tokamak Fusion Test Reactor (TFTR) magnetic environment. This implies that the magnetic impact was much worse compared to that of the TFTR operation, since the $\mathrm{OH}$ flux change was much stronger due to a short pulse length compared to the TFTR operation (1/10) but the plasma current is about the same for two machines. After this campaign, floor vibration was isolated by activating an air cushion system on the optical tables. During the 2002 campaign, two channels were successfully operated at tangencies of 32 and $57 \mathrm{~cm}$. Sample data of the measured electron density are shown in Fig. 6 together with the comparison of FIReTIP measurements with Thomson scattering measurements. This figure shows that the oscillations from the mechanical vibrations from $\mathrm{OH}$ impacts were further removed using homemade vibration isolation stands for the retroreflectors. The electron density mea- 


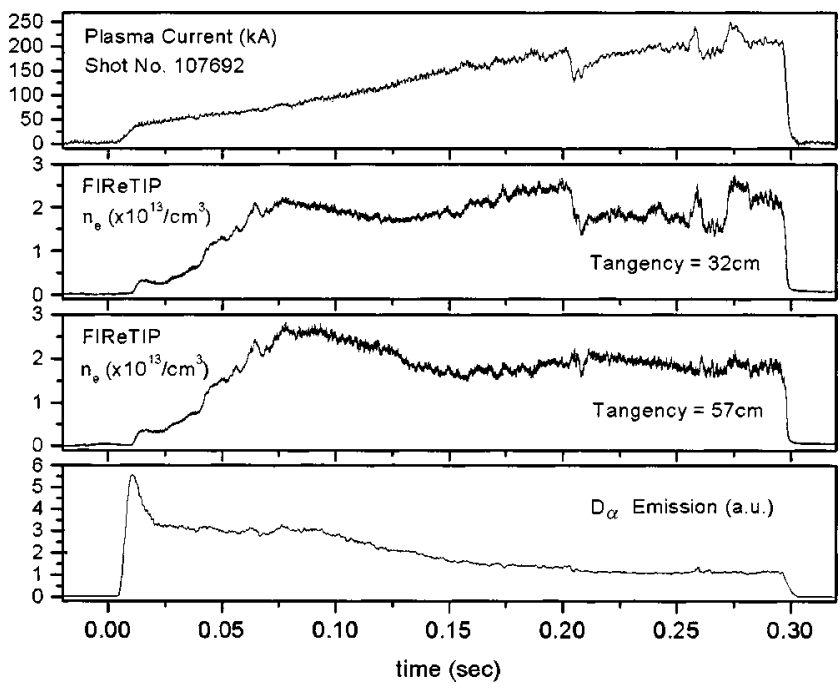

FIG. 7. Density evolution during the Coaxial Helicity Injection (CHI) for two measured channels are illustrated. Note the high and fast fluctuations in these traces.

surements showed excellent agreements with the Thomson scattering measurements after the installation of vibration free stands. Figure 7 is the first data of the electron density measurement for the coaxial helicity injection (CHI) plasma on NSTX that demonstrates a fast changing density evolution with fluctuations. An example of the Faraday rotation measurements from the polarimetry is shown in Fig. 8. Since the toroidal field current was constant and plasma beta was reasonably low, the Faraday rotation angles followed the electron density. The system resolution is suffering from small but coherent oscillations. Though they are not shown in the figure due to the smoothing, the amplitudes of these oscillations are indicated by error bars in Fig. 9 where it is shown that there is a reasonable agreement between the measured data and calculated Faraday rotation angles using both vacuum field and magnetic field via equilibrium (EFIT) code. The causes of these oscillations are currently under

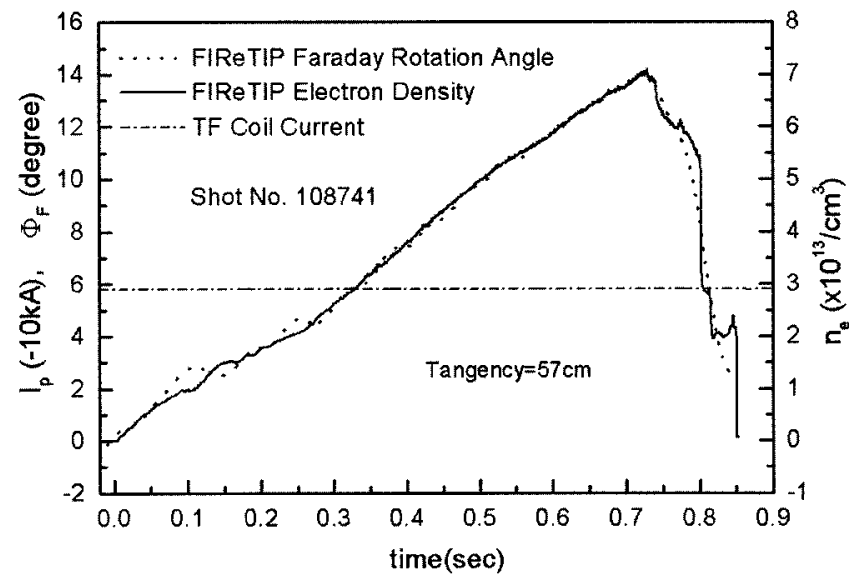

FIG. 8. Time history of Faraday rotation measurement is compared with the density traces (Faraday rotation data are smoothed by filtering out high frequency components).

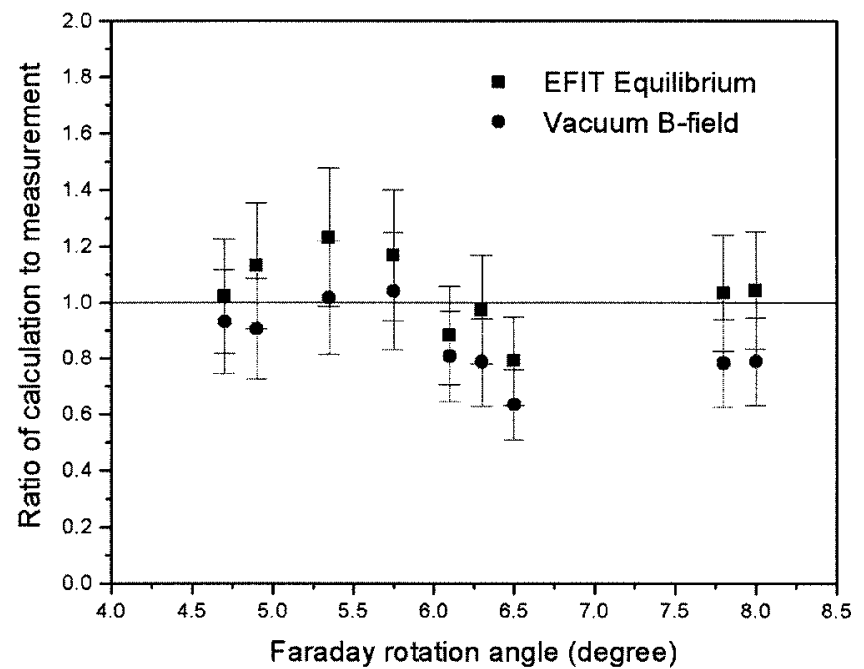

FIG. 9. Comparison between Faraday rotation data and calculated rotation angles using both vacuum magnetic field and magnetic field calculated by EFIT equilibrium code.

investigation in order to improve the quality of signal so that it can be useful to identify para and diamagnetic effects on spherical torus discharges.

\section{CONCLUSION}

A Stark-tuned optically pumped FIR laser was successfully applied to the plasma interferometry and polarimetry for the first time on NSTX. The significant increase of the modulation frequency allowed fast time resolution measurement in interferometry and polarimetry. Although the time resolution of the current system is limited to $\sim 100 \mathrm{kHz}$, the bandwidth of the system can be extended using the digital phase counting system used in the TFTR system $(0.25 \mathrm{MHz}$ bandwidth at $1 \mathrm{MHz}$ IF frequency). In addition to the improved time resolution, the higher modulation frequency provided more operational room for beat frequency drift which has been a common problem. Extensive work was done to isolate the system from mechanical vibrations originated from various sources and successfully produced vibration free density traces. Future tasks include efforts to expand the system into a full seven chord system for the 2D data of electron density and toroidal magnetic field.

\section{ACKNOWLEDGMENTS}

This work is supported by U.S. Department of Energy Grant No. DOE DE-FG03-99ER54518. Support from the NSTX team of PPPL is acknowledged. Thanks go to B. Leblanc and L. Roquemore for Thomson data and engineering support, respectively.

${ }^{1}$ D. K. Mansfield, P. A. Krug, M. Vocaturo, L. Guttadora, M. Rockmore, and K. Micai, Appl. Opt. 31, 5030 (1992).

${ }^{2}$ H. Park, C. W. Domier, W. R. Geck, and N. C. Luhmann, Jr., Rev. Sci. Instrum. 70, 710 (1999)

${ }^{3}$ J. H. Rommers, A. J. H. Donné, F. A. Karelse, and J. Howard, Rev. Sci. Instrum. 68, 1217 (1997).

${ }^{4}$ B. H. Deng, C. W. Domier, M. Johnson, K. C. Lee, B. R. Nathan, N. C. Luhmann, Jr., and H. Park, Rev. Sci. Instrum. (these proceedings).

${ }^{5}$ G. Bionducci, M. Inguscio, A. Moretti, and F. Strumia, Infrared Phys. 19, 297 (1979). 